\title{
A self-regularising DVM-PSE method for the modelling of diffusion in particle methods
}

\author{
Paul Mycek ${ }^{a, b}$, Grégory Pinon ${ }^{a, ~}$, Grégory Germain ${ }^{b}$, Élie Rivoalen ${ }^{c, a}$
}

\footnotetext{
a Laboratoire Ondes et milieux complexes, UMR 6294, CNRS-université du Havre, 53, rue de Prony, BP 540, 76058 Le Havre cedex, France

b IFREMER, Marine Structures Laboratory, 150, quai Gambetta, BP 699, 62321 Boulogne-Sur-Mer, France

c Laboratoire d'optimisation et fiabilité en mécanique des structures, EA 3828, INSA de Rouen, avenue de I'Université, BP 08, 76801 Saint-Étienne-du-Rouvray, France
}

\author{
*: Corresponding author : Grégory Pinon, tel.: +33 (0) 235217123 ; fax: +33 (0) 232744719 ; \\ email address : gregory.pinon@univ-lehavre.fr \\ paul.mycek@ifremer.fr ; gregory.germain@ifremer.fr ; elie.rivoalen@insa-rouen.fr
}

\begin{abstract}
:
The modelling of diffusive terms in particle methods is a delicate matter and several models have been proposed in the literature. The Diffusion Velocity Method (DVM) consists in rewriting these terms in an advective way, thus defining a so-called diffusion velocity. In addition to the actual velocity, it is used to compute the particles displacement. On the other hand, the well-known and commonly used Particle Strength Exchange method (PSE) uses an approximation of the Laplacian operator in order to model diffusion. This approximation is based on an exchange of particles strength.

Although DVM is particularly well suited to particle methods since it preserves their Lagrangian aspect, its major drawback stems in the fact that it suffers from severe singular behaviours. This paper intends to give insights and ideas for coping with these issues, based on an exact decomposition of the diffusion coefficient allowing a hybrid DVM-PSE treatment of diffusive terms.
\end{abstract}

Keywords : Particle method ; Diffusion ; DVM ; PSE 


\section{Introduction}

Particle methods, such as vortex methods or Smoothed Particle Hydrodynamics, generally consist of a Lagrangian transport, thanks to fluid dynamics equations, of quantitative information carried by particles. The transport equations linked to each particle (position, vortical intensity, concentration, temperature, etc.) are in fact partial differential equations. Owing to the Lagrangian aspect of the method, it is particularly well suited to flows where convective effects are dominant. However, transport equations may involve terms that do not represent advection. This is the case of the Navier-Stokes equations, which involve, for instance, a diffusion term.

The Diffusion Velocity Method (DVM) is a technique which consists in writing the diffusive term, with either uniform or variable diffusion coefficient, as a convective term. It was first introduced by Fronteau \& Combis [1] in 1984 and popularized by Degond \& Mustieles [2, 3], Ogami \& Akamatsu [4] and Kempka \& Strickland [5] in the early 1990s. This method was then largely analysed [6-10], adapted to dispersion equations [10-12], coupled with turbulence models $[13,14]$ and extended to the diffusion of a vector field and to axisymmetric flows [15-17].

DVM is particularly well suited to the resolution of diffusion or dispersion in open media. Indeed, there is an adaptation of the convected and diffused support, represented by the particles, in an extending domain. Applications concern for instance, pollutant dispersion in porous media [18], heat-vortex interaction [19], airfoil wake modelling [20], as well as the modelling of the diffusive term in the Navier-Stokes [15], heat [21] or Lotka-Volterra [22] equations.

However, DVM suffers from a severe singularity issue as the quantity to diffuse tends to zero. For instance, this is the case of the diffusion of two-dimensional counter-rotative vortices, for which the vorticity spatial distribution passes through zero. This problem has already been addressed, and partially satisfying or case-specific workarounds were proposed [6, 10, 19].

On the other hand, the Particle Strength Exchange (PSE) method [23-25], is based on a discrete approximation of the Laplacian operator and has a non-singular behaviour. It is today the most commonly used method for treating diffusion problems in particle methods. The main drawback of this method is that the domain does not extend since diffusion is simply modelled by exchanging strength on the particles supports. As a consequence, error might then appear at the domain boundary. A widely spread technique in particle methods to avoid this boundary error consists in remeshing the particles on a Cartesian grid $[26,27]$. This strategy is not investigated here, so as to study the purely Lagrangian behaviour of the particles and preserve the orders of approximation ensured by the kernel. However, it should be stated that remeshing is often unavoidable in more general and realistic configurations, in order to ensure that the particles always overlap. Alternatively, Schrader et al. [28] recently suggested to build the PSE kernel so as to correct the quadrature error by introducing the concept of discrete moments. Unfortunately, for non-uniformly distributed particles, this requires to re-compute the kernel for each particle at each time step, which is extremely time consuming and thus prohibitive.

This paper deals with the DVM singularity issues. It focuses on a critical test case in which the quantity to diffuse passes through zero with a steep gradient. Considerations about the elaboration of a self-regularising method are given, together with numerical computation results. This method consists in a hybrid DVM-PSE treatment which benefits only from the advantages of both methods.

\section{Regularised DVM-PSE (rDVM-PSE)}

The transport equation associated to a passive scalar $c(\boldsymbol{x}, t)$ is considered: 


$$
\frac{\partial c}{\partial t}+\nabla \cdot(\boldsymbol{u} c)=\nabla \cdot(\nu \nabla c)
$$

This transport equation can be written in a purely convective form, which leads to the classical DVM model [1-10]:

$$
\frac{\partial c}{\partial t}+\boldsymbol{\nabla} \cdot\left[\left(\boldsymbol{u}+\boldsymbol{u}_{d}\right) c\right]=0, \quad \text { with } \quad \boldsymbol{u}_{d}(\boldsymbol{x}, t)=-\nu \frac{\boldsymbol{\nabla} c}{c} .
$$

The diffusion coefficient $\nu$ can be split such that $\nu=\nu^{*}+\nu^{* *}$. Thus,

$$
\frac{\partial c}{\partial t}+\nabla \cdot\left[\left(\boldsymbol{u}+\boldsymbol{u}_{d}^{*}\right) c\right]=\nabla \cdot\left(\nu^{* *} \nabla c\right)
$$

with $\boldsymbol{u}_{d}^{*}=-\nu^{*} \nabla c / c$. A first decomposition of $\nu$ is considered by choosing $\nu^{*}$ as follows:

$$
\nu^{*}=\nu \frac{c^{2}}{c^{2}+c_{0}^{2}}
$$

with $c_{0}$ some non-zero constant homogeneous to $c$. By doing so, the regular behaviour of $\boldsymbol{u}_{d}^{*}$ is ensured. On the contrary, the DVM modelling of the diffusion induced by $\nu^{* *}$ would lead to a singular formulation. Therefore, the PSE method will be used for the modelling of this part of the diffusion. It should be noted that, in this formulation, $\nu^{* *}$ depends on the position, so the right-hand term does not reduce to a Laplacian and must be treated with a non-uniform coefficient [23].

The physical domain is discretised into $N$ particles of support $\mathcal{P}_{i}$ whose volume is $\sigma_{i}$, for $i$ going from 1 to $N$. For each particle, a position vector $\boldsymbol{X}_{i}(t)$ and a weight $C_{i}(t)$ are defined as follows:

$$
\boldsymbol{X}_{i}(t)=\frac{\int_{\mathcal{P}_{i}} \boldsymbol{x}(t) d \boldsymbol{x}}{\int_{\mathcal{P}_{i}} d \boldsymbol{x}} \quad \text { and } \quad C_{i}(t)=\int_{\mathcal{P}_{i}} c(\boldsymbol{x}, t) d \boldsymbol{x} \approx c\left(\boldsymbol{X}_{i}, t\right) \sigma_{i} .
$$

The discrete version of transport equations (3) then reads:

$$
\left\{\begin{array}{l}
\frac{d \boldsymbol{X}_{i}}{d t}=\boldsymbol{u}\left(\boldsymbol{X}_{i}, t\right)+\tilde{\boldsymbol{u}}_{d}^{*}\left(\boldsymbol{X}_{i}, t\right) \\
\frac{d C_{i}}{d t}=\sum_{j=1}^{N} \frac{\tilde{\nu}_{i}^{* *}+\tilde{\nu}_{j}^{* *}}{2}\left(C_{j} \sigma_{i}-C_{i} \sigma_{j}\right) \eta_{\varepsilon}^{l a p}\left(\boldsymbol{X}_{i}-\boldsymbol{X}_{j}\right)
\end{array}\right.
$$

with $\tilde{\boldsymbol{u}}_{d}^{*}\left(\boldsymbol{X}_{k}, t\right)=-\left.\tilde{\nu}_{k}^{*} \frac{\widetilde{\boldsymbol{\nabla}} c}{\tilde{c}}\right|_{k}, \quad \tilde{\nu}_{k}^{*}=\left.\nu \frac{\tilde{c}^{2}}{\tilde{c}^{2}+c_{0}^{2}}\right|_{k}, \quad \tilde{\nu}_{k}^{* *}=\left.\nu \frac{c_{0}^{2}}{\tilde{c}^{2}+c_{0}^{2}}\right|_{k}$ and where $\tilde{r}$ denotes the particle approximation. This formulation will be referred to as the rDVM-PSE model. The particle approximations of $c$ and of its gradient $\nabla c$ are basically given by:

$$
\left.\tilde{c}\right|_{k}=\sum_{i=1}^{N} C_{i}(t) \zeta_{\varepsilon}\left(\boldsymbol{X}_{k}-\boldsymbol{X}_{i}\right), \text { and }\left.\widetilde{\nabla} c\right|_{k}=\sum_{i=1}^{N} C_{i}(t) \boldsymbol{\nabla} \zeta_{\varepsilon}\left(\boldsymbol{X}_{k}-\boldsymbol{X}_{i}\right)
$$

where $\zeta$ is an interpolation kernel [29] of any given order of accuracy. In the sequel, the results shown were obtained using a $6^{\text {th }}$ order Gaussian kernel [25].

In the rDVM-PSE formulation, one can notice that $\nu^{*}$ tends to zero (and thus $\nu^{* *}$ tends to $\nu$ ) when $c$ tends to zero. This is promising because it means that the DVM method will not be used when $c$ tends to zero. Conversely, $\nu^{*}$ tends to $\nu$ when $c$ tends to infinity, and more generally, $\nu^{*} / \nu$ is significantly higher than $\nu^{* *} / \nu$ as soon as $c$ gets significantly higher than $c_{0}$.

The DVM treated part $\boldsymbol{u}_{d}^{*}$ corresponds to the regularised version of DVM proposed by Lions \& MasGallic [10]. However, their formulation does not take the $\nu^{* *}$ part into account, which introduces an error in the model depending on the value of $c_{0}$, which may be seen as a regularising parameter $\varepsilon$ and which should thus be a significantly small value. In addition, this regularisation alone does not prevent the 
particles from crossing zero in the case of steep gradient (see the test case presented in section 4 ). On the contrary, in the above presented model (3) with $\boldsymbol{u}_{d}^{*}$ chosen as in (4), $c_{0}$ is a transition parameter between DVM and PSE and thus does not need to tend to zero. It also ensures a regular behaviour as $c$ tends to zero as in [10].

The singularity issues inherent to DVM are avoided, but accuracy problems may occur with PSE at the domain boundary, due to the lack of domain expansion.

\section{Weighted regularised DVM-PSE (wrDVM-PSE)}

The main idea in the previous hybrid formulation resides in the decomposition of $\nu$ into two complementary diffusion coefficients $\nu^{*}$ and $\nu^{* *}$. In the previous formulation, the diffusion induced by $\nu^{*}$ corresponded to the regularised DVM formulation suggested by [10]. However, it is clear that any other exact decomposition of $\nu$ might be appropriate. Equations (6) thus describe the generic numerical scheme with $\nu^{*}$ the DVM-treated and $\nu^{* *}$ the PSE-treated diffusion coefficients provided that $\nu=\nu^{*}+\nu^{* *}$.

From this statement, relevant decompositions should be obtained by ensuring that the diffusion coefficient part that induces the DVM-treated diffusion tends to zero in and only in singular zones. Equation (8) may correspond to such a decomposition:

$$
\nu^{*}=\nu \frac{(c / w)^{2}}{(c / w)^{2}+c_{0}^{2}},
$$

with

$$
w(x)=\sum_{j ; c(x) c\left(X_{j}\right) \leq 0} \frac{1}{\lambda} W\left(\frac{x-X_{j}}{\lambda}\right) \sigma_{j},
$$

where $W$ is a non-negative interpolation function in one dimension. For instance, $W$ can be a kernel with non-compact support such as Gaussian kernels, or a kernel with compact support such as $M_{3}, M_{4}$ or truncated Gaussian kernels [27]. Since $w$ is dimension-free, $c / w$ and $c_{0}$ are homogeneous to $c$.

This formulation is close to formulation (6), with the difference that quantity $c$ is now "weighted" by $w$. The purpose of $w$ is to assess the closeness to any area where concentration $c$ passes through zero. As a matter of fact, from equation (9), one can see that the value of $w$ gets higher as the location where it is evaluated gets closer to a zero-crossing area. This formulation can be rewritten as follows:

$$
\nu^{*}=\nu \frac{c^{2}}{c^{2}+c_{w}^{2}(x)}
$$

with $c_{w}(x)=c_{0} w(x)$. One can then see the weighting process differently: the weight applies to the transition parameter and eventually leads to a real adaptive concentration parameter $c_{w}(x)$. This means that the closer to zero crossing zones, the higher the transition parameter and thus the less the DVM method tends to be used.

It is important to notice that this formulation has the advantage of being rather general: as a matter of fact, depending on the problem, one may chose any appropriate weighting function $w$.

The one-dimensional smoothing kernel $W$ used in the following results is a $2^{\text {nd }}$ order Gaussian kernel, defined by $W(r)=e^{-r^{2}} / \sqrt{\pi}$. 


\section{The double Gaussian test case}

A one-dimensional double Gaussian test case is presented, in which the analytic solution $c(x, t)$ passes through zero at $x=0$ for any $t>0$ :

$$
c(x, t)=\frac{1}{\sqrt{4 \pi \nu t}}\left[\exp \left(-\frac{(x-1)^{2}}{4 \nu t}\right)-\exp \left(-\frac{(x+1)^{2}}{4 \nu t}\right)\right]
$$

This corresponds to the solution of the pure diffusion equation $\partial c / \partial t=\nu \partial^{2} c / \partial x^{2}$ with the following initial condition, for all $x \in \mathbb{R}$ :

$$
c(x, 0)=\delta(x-1)-\delta(x+1)
$$

where $\delta$ denotes the Dirac measure.

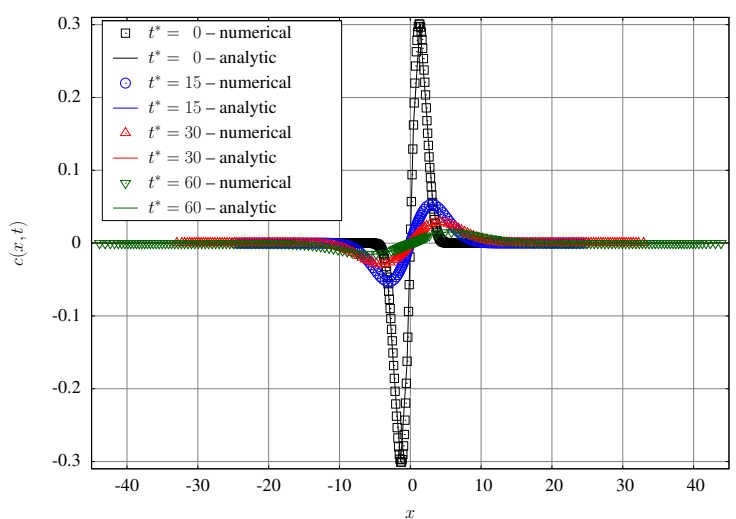

(a) $\left(c_{0}=3.0, \lambda=1.5\right)$

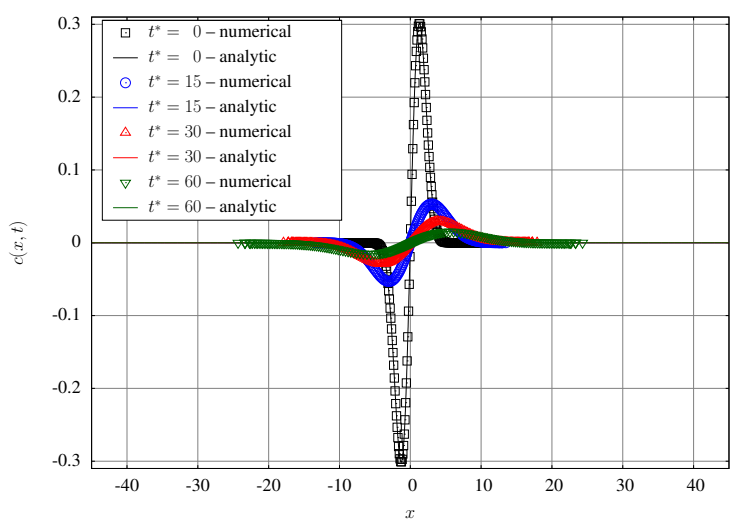

(c) $\left(c_{0}=1.0, \lambda=3.5\right)$

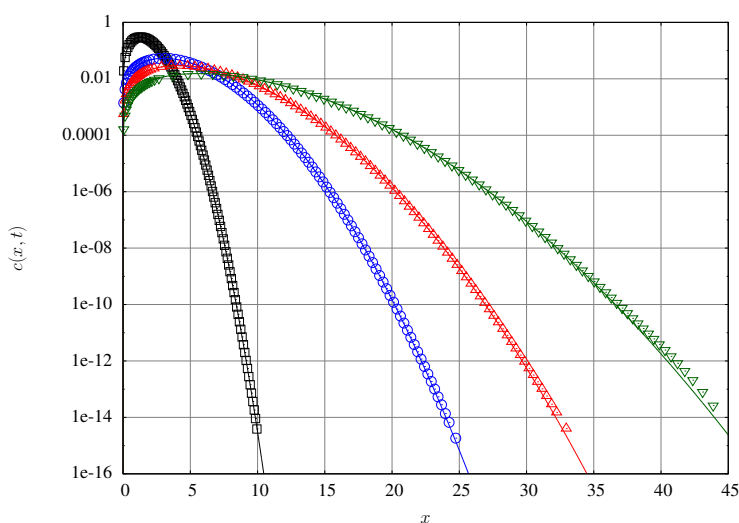

(b) $\left(c_{0}=3.0, \lambda=1.5\right)$, log. scale

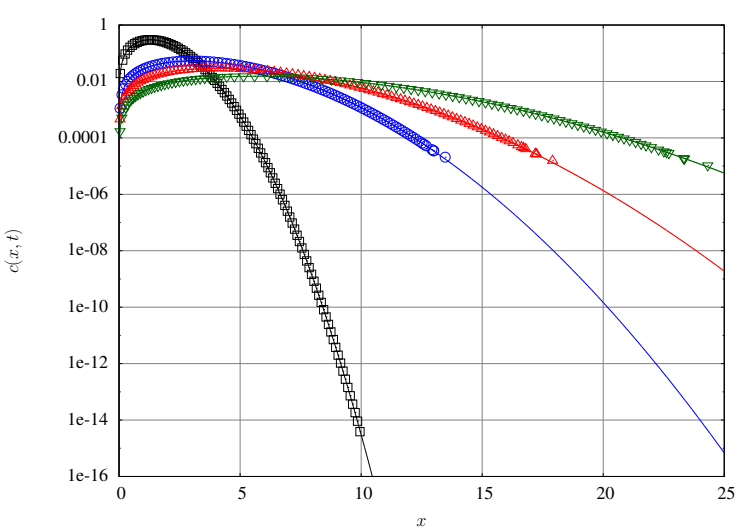

(d) $\left(c_{0}=1.0, \lambda=3.5\right)$, log. scale

Figure 1. Concentration and particles position evolution over different instants $t^{*}=t-t_{0}$ with $6^{\text {th }}$ order wrDVM-PSE, $N=200$ particles and $10^{-3}$ as the numerical time step. Two different pairs of parameters $\left(c_{0}, \lambda\right)$ are chosen: $(3.0,1.5)($ top) and $(1.0,3.5)$ (bottom).

Computations were run with different sets of parameters $\left(c_{0}, \lambda\right)$. Amongst them, two chosen pairs of parameters are shown in the sequel: $(3.0,1.5)$ and $(1.0,3.5)$. Figure 1 shows the concentration distribution 
and the particles' position at different instants obtained using a $6^{\text {th }}$ order wrDVM-PSE model (i.e. with $\zeta$ being a $6^{\text {th }}$ order kernel) with those pairs of parameters. The initial time $t_{0}$ and the diffusion coefficient $\nu$ are chosen such that $4 \nu t_{0}=2.5$ and the initial domain length is $L=20$ discretised with 200 particles. The numerical time-step is $10^{-3}$.

Both computations give good results, even after $t^{*}=t-t_{0}=60$. The difference between the two computations resides in the amount of diffusion velocity, in other words in how much DVM is counterbalanced with PSE, especially at the boundaries. As a matter of fact, as show Figures 1a and 1c, the domain does not extend as far with the second pair of parameters $(1.0,3.5)$ as it does with the first one $(3.0,1.5)$. This can be explained by the distribution of the DVM proportion $\nu^{*} / \nu$, depending on the parameters $c_{0}$ and $\lambda$.

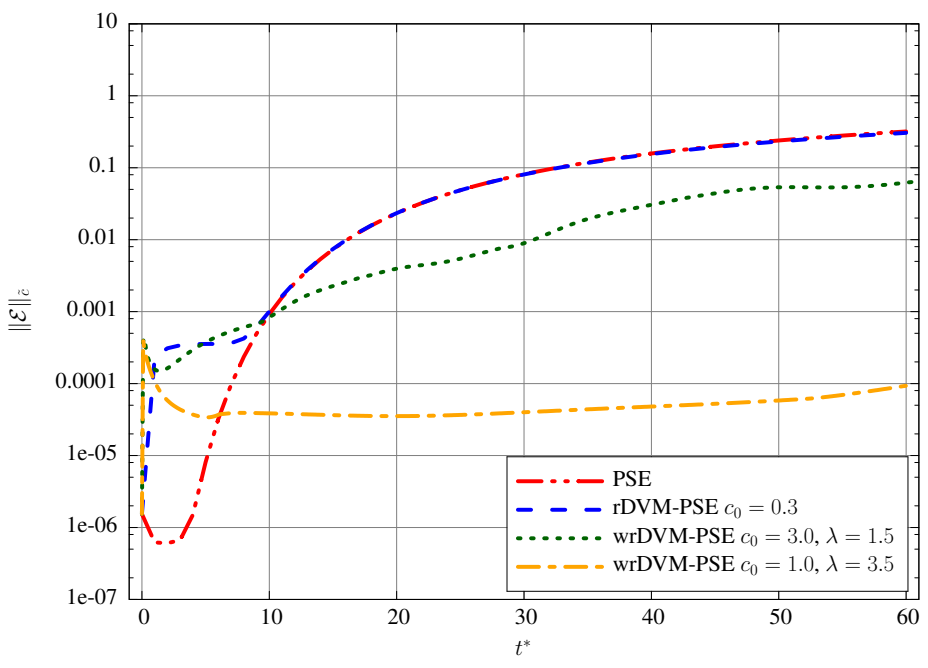

Figure 2. Concentration $L_{2}$ error as a function of time for the double Gaussian case. Comparison between PSE (red dashed-double-dotted line), rDVM-PSE with $c_{0}=0.3$ (blue dashed line), wrDVM-PSE with $c_{0}=3.0$ and $\lambda=1.5$ (green dotted line) and wrDVM-PSE with $c_{0}=1.0$ and $\lambda=3.5$ (orange dashed-dotted line). Computations were run using a $6^{\text {th }}$ order approximation and $N=200$ particles.

The fact that the second pair of parameters $(1.0,3.5)$ gives better results in terms of concentration error is highlighted by Figure 2 which presents the evolution of the concentration $L_{2}$ error as a function of time for different models able to treat the double Gaussian case. The first observation is that rDVM-PSE gets finally equivalent to PSE in terms of $L_{2}$ error, since the increasing error at the boundaries rapidly accounts for the major part of the overall error. Second, wrDVM-PSE with the first pair of parameters $(3.0,1.5)$ gives more satisfying results but the overall error still follows a PSE-like evolution (not for the same reasons, though). Eventually, wrDVM-PSE with the second pair of parameters $(1.0,3.5)$ gives the best results in terms of concentration $L_{2}$ error, which remains rather low and constant over time.

\section{Conclusion}

This paper intended to suggest some ideas for the regularisation of the original DVM formulation. The proposed method, namely wrDVM-PSE, shows satisfying results. Unfortunately, it remains very sensitive to the choice of $c_{0}$ and $\lambda$, which is a limit to any generic use of this model. The exact decomposition of the diffusion coefficient is nevertheless a promising idea in the prospect of coping with zero-crossing 
singularities. Future work should lead to more general models based on this decomposition, which could then become less case-specific. Additional studies should now be carried out to improve and stabilize this method.

\section{Acknowledgements}

The authors would like to thank Haute-Normandie Regional Council and IFREMER for their financial support of co-financed $\mathrm{PhD}$ theses, as well as the RHYNO project. The authors also thank the CRIHAN for their available numerical computation resources.

\section{References}

[1] J. Fronteau, P. Combis, A lie admissible method of integration of folkler-plank equations with non linear coefficients (exact and numerical solutions), Hadronic J. 7 (1984) 911-930.

[2] F.-J. Mustieles, L'équation de Boltzmann des semiconducteurs. Étude mathématique et simulation numérique, Ph.D. thesis, École Polytechnique, 1990.

[3] P. Degond, F.-J. Mustieles, A deterministic approximation of diffusion equations using particles, SIAM Journal on Scientific and Statistical Computing 11 (1990) 293-310.

[4] Y. Ogami, T. Akamatsu, Viscous flow simulation using the discrete vortex model - the diffusion velocity method, Computers \& Fluids 19 (1991) 433-441.

[5] S. Kempka, J. Strickland, A method to simulate viscous diffusion of vorticity by convective transport of vortices at a non-solenoidal velocity, Technical Report SAND-93-1763, Sandia Laboratory, 1993. URL: http://www.osti.gov/energycitations/servlets/purl/10190654-dLAMwy/native/.

[6] J. H. Strickland, S. N. Kempka, W. P. Wolfe, Viscous diffusion of vorticity using the diffusion velocity concept, ESAIM: Proc. 1 (1996) 135-151.

[7] S. Mas-Gallic, A presentation of the diffusion velocity method, in: P. Leach, S. Bouquet, J.-L. Rouet, E. Fijalkow (Eds.), Dynamical Systems, Plasmas and Gravitation, volume 518 of Lecture Notes in Physics, Springer Berlin / Heidelberg, 1999, pp. 74-81. URL: http://dx.doi.org/10.1007/BFb0105914, 10.1007/BFb0105914.

[8] G. Lacombe, S. Mas-Gallic, Presentation and analysis of a diffusion-velocity method, ESAIM: Proc. 7 (1999) 225-233.

[9] G. Lacombe, Analyse d'une équation de vitesse de diffusion, Comptes Rendus de l'Académie des Sciences - Series I - Mathematics 329 (1999) 383 - 386.

[10] P.-L. Lions, S. Mas-Gallic, Une méthode particulaire déterministe pour des équations diffusives non linéaires, Comptes Rendus de l'Académie des Sciences - Series I - Mathematics 332 (2001) 369-376.

[11] A. Chertock, D. Levy, Particle methods for dispersive equations, Journal of Computational Physics $171(2001) 708-730$.

[12] A. Chertock, D. Levy, A particle method for the KdV equation, J. Sci. Comput. 17 (2002) 491-499.

[13] R. Milane, S. Nourazar, On the turbulent diffusion velocity in mixing layer simulated using the vortex method and the subgrid scale vorticity model, Mechanics Research Communications 22 (1995) 327 -333 .

[14] R. E. Milane, Large eddy simulation (2d) using diffusion-velocity method and vortex-in-cell, International Journal for Numerical Methods in Fluids 44 (2004) 837-860. 
[15] E. Rivoalen, S. Huberson, F. Hauville, Simulation numérique des équations de Navier-Stokes 3D par une méthode particulaire, Comptes Rendus de l'Académie des Sciences - Series IIB - MechanicsPhysics-Chemistry-Astronomy 324 (1997) 543-549.

[16] J. Grant, J. Marshall, Diffusion velocity for a three-dimensional vorticity field, Theoretical and Computational Fluid Dynamics 19 (2005) 377-390. 10.1007/s00162-005-0004-8.

[17] E. Rivoalen, S. Huberson, Numerical simulation of axisymmetric viscous flows by means of a particle method, Journal of Computational Physics 152 (1999) 1 - 31.

[18] A. Beaudoin, S. Huberson, E. Rivoalen, Simulation of anisotropic diffusion by means of a diffusion velocity method, Journal of Computational Physics 186 (2003) 122 - 135.

[19] Y. Ogami, Simulation of heat-vortex interaction by the diffusion velocity method, ESAIM: Proc. 7 (1999) 313-324.

[20] S. Guvernyuk, G. Dynnikova, Modeling the flow past an oscillating airfoil by the method of viscous vortex domains, Fluid Dynamics 42 (2007) 1-11.

[21] Y. Dynnikov, G. Dynnikova, Numerical stability and numerical viscosity in certain meshless vortex methods as applied to the navier-stokes and heat equations, Computational Mathematics and Mathematical Physics 51 (2011) 1792-1804.

[22] G. Gambino, M. Lombardo, M. Sammartino, A velocity-diffusion method for a lotka-volterra system with nonlinear cross and self-diffusion, Applied Numerical Mathematics 59 (2009) 1059 - 1074.

[23] P. Degond, S. Mas-Gallic, The weighted particle method for convection-diffusion equations. Part I: The case of an isotropic viscosity, Math. Comp. 53 (1989) 485-507.

[24] J. Choquin, S. Huberson, Particles simulation of viscous flow, Computers \& Fluids 17 (1989) 397 410.

[25] J. D. Eldredge, A. Leonard, T. Colonius, A general deterministic treatment of derivatives in particle methods, Journal of Computational Physics 180 (2002) 686-709.

[26] G. Cottet, P. Koumoutsakos, Vortex methods: theory and practice, Cambridge University Press, 2000.

[27] J. Monaghan, Extrapolating B splines for interpolation, Journal of Computational Physics 60 (1985) $253-262$.

[28] B. Schrader, S. Reboux, I. F. Sbalzarini, Discretization correction of general integral pse operators for particle methods, Journal of Computational Physics 229 (2010) 4159-4182.

[29] J. T. Beale, A. Majda, High order accurate vortex methods with explicit velocity kernels, Journal of Computational Physics 58 (1985) 188-208. 